\title{
Für alle Zeiten und Culturen! Das revidierte Internationale System der Einheiten
}

\author{
Joachim Hermann Ullrich ${ }^{1}$ \\ ${ }^{1}$ Physikalisch-Technische Bundesanstalt, Bundesallee 100, 38116 Braunschweig, Deutschland
}

\section{Zusammenfassung}

Der 20. Mai 2019 markiert eine Zäsur in der Entwicklung der physikalischen und technischen Maßeinheiten. An diesem Tag, der zugleich der Weltmetrologietag des Jahres 2019 ist, trat ein Paradigmenwechsel im Internationalen System der Einheiten (Système international d'unités, kurz: SI) in Kraft. Vorbei die Zeiten, in denen ein Metallzylinder definiert, was ein Kilogramm sein soll, oder eine spezifisch herzustellende Isotopenmischung von Wasser die Einheit der Temperatur festlegt. Naturkonstanten, darunter die Lichtgeschwindigkeit $c$, das Planck'sche Wirkungsquantum $h$ und die BoltzmannKonstante $k$, definieren ab diesem Zeitpunkt alle physikalischen Einheiten. Das so definierte Einheitensystem bildet eine Art "universelle Sprache" und hat einzigartige, insbesondere auch technologisch relevante Eigenschaften: Die Naturkonstanten bevorzugen, anders beispielsweise als das Urkilogramm oder der Tripelpunkt von Wasser, keine speziellen Punkte auf der jeweiligen Einheitenskala, so dass viele Einheiten nun entlang der gesamten Skala realisiert werden können. Zum anderen ist das revidierte SI offen für technologische Innovationen bei der Realisierung der Einheiten: jede passende Gleichung der Physik kann zur Realisierung herangezogen werden und die Genauigkeit der Realisierung kann entsprechend des technischen Fortschritts gesteigert werden, ohne Begrenzung durch die Definition selbst.

Keywords: Metrologie, Internationales Einheitensystem, Meterkonvention, Naturkonstanten

\section{Die Anfänge des Messens}

Schon in den alten Hochkulturen wussten die Menschen, dass Eigenschaften von Körpern, Substanzen und Phänomenen gemessen werden können. Das Prinzip des Messens lautete damals wie heute: Um das Ergebnis einer Messung auszudrücken, braucht man eine Zahl und eine Einheit. Die Einheit ist typischerweise durch ein Artefakt oder verkörpertes Normal festgelegt, das einen speziellen Wert der Messgröße repräsentiert.

Das berühmteste (und letzte) Artefakt ist das Urkilogramm, das 1889 von der Meterkonvention als die Einheit für die Masse definiert worden war. Im November 2018 entschied die 26. Generalkonferenz für Maße und Gewichte (CGPM), das Urkilogramm abzulösen und gleichzeitig drei weitere Basiseinheiten neu zu definieren. Diese Entwicklung kann man als Konsequenz der Tatsache sehen, dass die Natur im Kleinsten "quantisiert" ist und durch die Quantenmechanik beschrieben wird. Quanten sind die kleinsten natürlich vorkommenden Einheiten für bestimmte Größen. So kann man einen elektrischen Strom messen, indem man in einem Leiter fließende, einzelne Elektronen, also die kleineste Einheit der elektrischen Ladung, in einer vorgegebenen Zeit zählt und so "das neue Ampere" realisiert.
Von Artefakten zu Quanten - mit diesen beiden Schlagworten lässt sich die generelle Entwicklung bei den Einheiten umreißen. Schon Maxwell und Planck forderten vor mehr als 100 Jahren, die Artefakte als Grundlage der Einheiten abzuschaffen. Insbesondere Planck schlug das ultimative Einheitensystem vor, das auf den - soweit heute experimentell nachgewiesen - unverrückbaren Naturkonstanten beruhen sollte. Diesen Weg haben die Metrologen, die Wissenschaftler, die sich mit der Messkunst beschäftigen, inzwischen höchst erfolgreich beschritten.

\section{Die ersten einheitlichen Einheiten}

Zunächst ein Blick zurück zur Französischen Revolution, die auch beim Thema Messen grundlegende Änderungen hervorbrachte. Das Ziel der Revolutionäre lautete, neue Einheiten zu schaffen, die für alle Zeiten und für alle Völker gelten sollten: à tous les temps, à tous les peuples. Die neuen Einheiten sollten nicht einem bestimmten Kaiser, einem bestimmten König oder einem bestimmten Land zugeordnet werden, sondern sie sollten allen Menschen auf der Welt gehören. Und sie sollten auf dem Dezimalsystem beruhen. Selbst der Tag wurde zunächst in zehn Stunden eingeteilt. Hier kehrte man allerdings nach kurzer 
Zeit zum alten Zwölfersystem der Babylonier zurück, das bis heute bei der Zeitmessung gilt. Als Grundlage der Einheiten sollte keine menschliche Eigenschaft mehr gelten, sondern die Eigenschaften der Erde. Dazu bildete die Académie des Sciences im Jahr 1790 ein Gremium, dem unter anderem Joseph-Louis Lagrange (Mathematiker und Astronom) und Pierre-Simon Laplace (Mathematiker, Physiker und Astronom) angehörten.

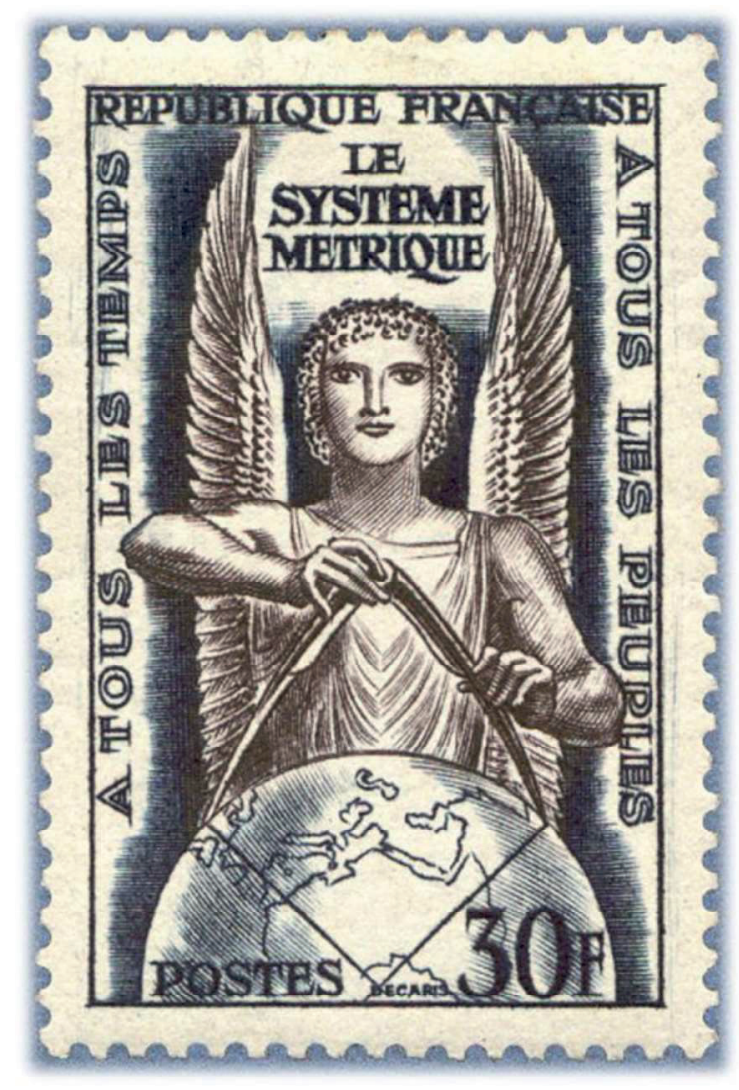

Abb.1: Diese Briefmarke illustriert die große Bedeutung der "metrischen Idee" und der neuen Einheit Meter, die von der Größe der Erde abgeleitet war. (Quelle: Musée de la Poste)

Dieses Gremium definierte die neue Längeneinheit als den vierzigmillionsten Teil eines Erdmeridians. Die Astronomen Jean-Baptiste Delambre und Pierre-François Méchain bekamen den Auftrag, einen Teil des Erdmeridians, der durch Paris lief, genau zu vermessen. Mitten in den Revolutionswirren triangulierten die beiden die Strecke von Dünkirchen bis Barcelona. Damit war die neue Längeneinheit Meter geschaffen. Da die Realisierung des Meters durch erneutes Vermessen zu aufwendig gewesen wäre, zog man sich wieder auf das Konzept der Maßverkörperung zurück und goss aus Platin ein Artefakt, das "Mètre des Archives". Die neue Einheit sowie das ganze neue, dezimale Messsystem sollten allgemein und universal gültig werden. Tatsächlich gelang dies aber erst fast hundert Jahre später.

\section{Die Meterkonvention}

Im Jahre 1875 führte das Thema „Einheiten“ 17 Staaten, darunter die meisten der damals wichtigsten Industriestaaten, in einem der ersten internationalen Verträge zusammen: der Meterkonvention. Interessanterweise waren die USA dabei, das Vereinte Königreich jedoch nicht. Es hatte seine eigenen Einheiten und war (als Commonwealth) groß genug, um eigene Wege zu gehen. Heute ist es Mitglied der Meterkonvention.

Zur Meterkonvention gehören mehrere Organe: die "Generalkonferenz für Maße und Gewichte" (CGPM) tagt alle vier Jahre und ist allein beschlussfähig. Die laufenden Geschäfte übernehmen das „Internationale Komitee für Maße und Gewichte" (CIPM) sowie diverse Konsultativkomitees (CC).

Mit der Unterzeichnung des Vertrages von 1875 akzeptierten alle beteiligten Staaten die neue Einheit Meter und erhielten 1889 Kopien des sogenannten Urmeters oder Internationalen Meterprotoypen, eines Präzisions-Strichmaßes gefertigt aus einer Legierung von Platin und Iridium, mit der Länge des „Mètre des Archives". Aus dem Meter abgeleitet wurde das Kilogramm als die Masse eines Kubikdezimeters Wasser bei 4 Grad Celsius definiert. Die Mitgliedsstaaten erhielten eine Kopie des Urkilogramm-Stückes, ebenfalls aus PtIr gefertigt. Der Urmeter und das Urkilogramm wurden in einem neugegründeten wissenschaftlichen Institut in Paris, dem „Internationalen Büro für Maße und Gewichte" (BIPM), das neben der Generalkonferenz und dem internationalen Komitee das dritte Organ der Meterkonvention ist, aufbewahrt und waren fortan für die Mitglieder die gültigen Referenznormale für Länge und Masse.

Jetzt konnte man alle Längenmessmittel oder Gewichtsstücke direkt oder über mehrere Zwischenschritte mit den nationalen Normalen vergleichen. In der Metrologie wird dies „metrologische Rückführung" genannt. Sie sorgt dafür, dass in allen Mitgliedsstaaten der Meterkonvention ein einheitliches Maßsystem verwendet wurde, und ist bis heute ein sehr erfolgreiches Prinzip.

Die Einheit der Zeit, die Sekunde, stand nicht unter der Verantwortung der Meterkonvention, sondern hier war bis zum Jahr 1967 die International Astronomical Union zuständig.

Alle drei Einheiten, Meter, Kilogramm und Sekunde, beruhten entsprechend der Vision der französischen Revolutionäre auf den Eigenschaften der Erde - entweder auf ihren Ab- 
messungen oder ihrer Rotation. Allerdings hatten damals schon einige Vordenker weiter gedacht: Der Physiker James Clerk Maxwell hatte schon 1870 gemahnt, die so definierten Einheiten seien keine wahren Invarianten, denn „die Eigenschaften unseres Planeten können sich ändern, und es wäre immer noch unser Planet, aber wenn sich die Eigenschaften eines Atoms änderten, wäre es nicht mehr dasselbe Atom." Daher sei es besser, die Eigenschaften von Atomen oder Molekülen als Grundlage für die Definition der Einheiten zu nehmen.

\section{Physikalisch-Technische Reichsanstalt}

Etwa zur gleichen Zeit, 1887, wurde in Berlin das weltweit erste Metrologieinstitut gegründet: die Physikalisch-Technische Reichsanstalt (PTR), die direkte Vorgängerin der heutigen Physikalisch-Technischen Bundesanstalt (PTB). Werner von Siemens und Hermann von Helmholtz waren neben Wilhelm Förster, dem Direktor der Normal-Eichungs-Kommission, der obersten Eichbehörde des Deutschen Reichs, die beiden Gründungsväter. Helmholtz wurde der erste Präsident der PTR. Er galt als einer der prominentesten Wissenschaftler seiner Zeit, Werner von Siemens war einer der prominentesten Unternehmer. Und genau in diesem Spannungsfeld - zwischen Wissenschaft und Industrie - bewegt sich die PTB heute noch; sie betreibt Wissenschaft in der Messtechnik für Gesellschaft, Industrie und nicht zuletzt als Grundlagenforschung.

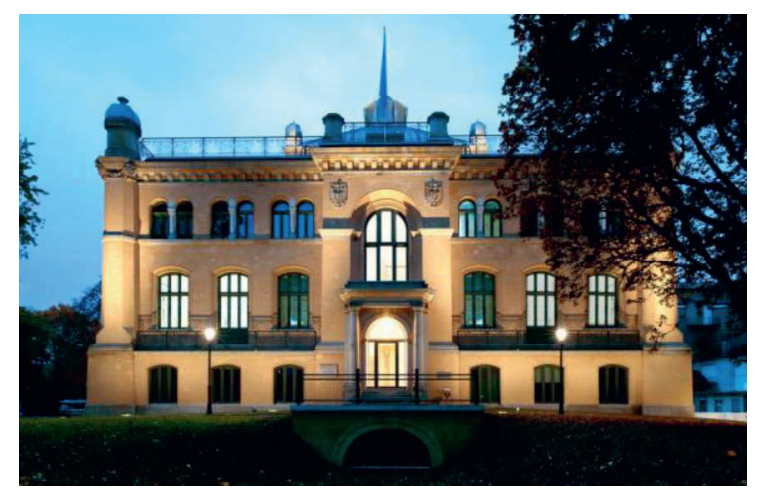

Abb. 2: Das Observatorium auf dem Gelände der ehemaligen PhysikalischTechnischen Reichsanstalt (PTR) in Berlin-Charlottenburg, dem heutigen Institut Berlin der PTB. (Quelle: PTB)

Das sogenannte Observatorium, das heute noch restauriert auf dem historischen Gelände in Berlin-Charlottenburg in der Nähe des ErnstReuter-Platzes steht, war das erste Laborgebäude der PTR und gleichzeitig das erste Großlabor im Deutschen Reich. Werner von
Siemens war der festen Überzeugung, dass es ein staatliches Institut brauche, das Präzisionsmessungen insbesondere auch für die elektrischen Einheiten durchführt und ganz allgemein die Einheitlichkeit des Messwesens in Deutschland sicherstellt. Da die Politik aus seiner Sicht nicht schnell genug vorankam, unterstützte er die Gründung der PTR mit seinem privaten Geld und baute insbesondere das Observatorium mit auf.

In diesem Gebäude fanden damals Messungen statt, die letztlich zur Entdeckung der Quantenmechanik führten. An einem sogenannten Schwarzen Strahler untersuchten Otto Lummer und seine Kollegen die Zusammenhänge zwischen Farbe und Intensität des Lichts. Sie fanden kleinste, unerklärliche $A b-$ weichungen von der damaligen theoretischen Vorhersage. Lummer rief noch am selben Abend Max Planck an, der später PTR-Kurator wurde. Planck konnte das Problem nur lösen, indem er in einem „Akt der Verzweiflung" (wie er selber sagte) annahm, die Energie der Strahlung werde nicht kontinuierlich, sondern in kleinen Päckchen transportiert, die er Quanten nannte. Seine Formel wurde später weltberühmt; die Konstante, die er dafür einführte, heißt seitdem Planck-Konstante. Somit wurde aus einer zunächst nicht breit beachteten Untersuchung an der PTR letztlich die Geburt der Quantenmechanik, die heute mit all ihren verschiedenen Facetten zu etwa 60 Prozent der Weltwirtschaft beiträgt.

\section{Natürliche Maßeinheiten}

Max Planck, im Kuratorium der Reichsanstalt tätig und damit in Gedanken den Einheiten nahe, sinnierte 1900 in seiner ebenfalls berühmt gewordenen Veröffentlichung zur Strahlung des schwarzen Körpers in der Zeitschrift „Annalen der Physik“: „Demgegenüber dürfte es nicht ohne Interesse sein zu bemerken, dass mit Zuhülfenahme der beiden auftretenden Constanten a und b (Anmerkung: eine war seine später sogenannte Planck-Konstante, die andere die Boltzmann-Konstante) die Möglichkeit gegeben ist, Einheiten für Länge, Masse, Zeit und Temperatur aufzustellen, welche, unabhängig von speciellen Körpern oder Substanzen, ihre Bedeutung für alle Zeiten und für alle, auch ausserirdische und aussermenschliche Culturen notwendig behalten und welche daher als ,natürliche Maaßeinheiten' bezeichnet werden können." Das waren im Jahr 1900 äußerst visionäre Gedanken.

Genau diesen Weg haben die Metrologen nun, mehr als hundert Jahre später, vollendet. Allerdings verlief er nicht ganz so scharf und konsequent, wie es Planck für die Einheiten für Länge, Zeit, Masse und Temperatur gefordert 
hat. Planck definierte sie mithilfe von Konstanten, von denen sich die meisten im gerade beschlossenen revidierten Einheitensystem wiederfinden, mit der Lichtgeschwindigkeit, seiner eigenen Planck-Konstanten, der Boltzmann-Konstanten sowie der (im heutigen SI nicht verwendeten) Gravitationskonstante. Doch die so definierten Einheiten sind extrem klein, die Planck-Zeit zum Beispiel beträgt nur etwa $5 \cdot 10^{-44}$ Sekunden. Eine so kleine Größe ist in der Praxis nicht gut verwendbar. Für theoretische und Hochenergie-Physiker sind die Planck-Einheiten dagegen sehr interessant und werden bis heute genutzt. Sie zeigen uns die Grenzen des heutigen Wissens auf, die genau bei dieser Planck-Zeit nach dem Urknall liegen: Was davor war, davon wissen wir so gut wie nichts, dort versagen unsere physikalischen Gesetze.

\section{Das Internationale Einheitensystem}

Das Internationale Einheitensystem SI (Système International d'unités) wurde 1960 von der Generalkonferenz für Maße und Gewichte eingeführt. Es beruhte bis zu seiner Revision am 20. Mai 2019 auf den sieben Basiseinheiten für Länge, Masse, Zeit, elektrische Stromstärke, Temperatur, Stoffmenge und Lichtstärke, von denen alle anderen Einheiten abgeleitet wurden, und bot einen Satz kohärenter Einheiten, um alles Erdenkliche zu messen, was von wissenschaftlicher, wirtschaftlicher, gesundheitlicher oder sonstiger Relevanz ist.

Das SI ist weltweit gültig. Denn inzwischen hat die Meterkonvention 102 Mitglieds- und assoziierte Länder, vier internationale Organisationen sowie 153 sogenannte Designierte Institute. Sie umfassen etwa 98 Prozent der gesamten Weltwirtschaft. Zusammengenommen ist dies eine globale Infrastruktur und damit die eigentliche Grundlage für globalen Handel. Weltweit sind etwa 65000 Prüf- und Kalibrierlaboratorien nach der internationalen Norm ISO/IEC 17025 akkreditiert. Damit haben sie sich verpflichtet, alle Messungen auf das SI rückzuführen - eine wichtige Voraussetzung dafür, um auf verschiedenen Kontinenten zu produzieren und international Handel zu treiben.

Dieses System wurde in der Folge kontinuierlich an neueste wissenschaftliche Erkenntnisse und technische Entwicklungen angepasst und diese Anpassungen für die Sekunde, den Meter und später das Ampere zeigen die Richtung zur jetzigen grundlegenden Revision auf. Das soll ein Blick auf die "Vorreiter" Sekunde und Meter erläutern:

Bei jeder Uhr gibt es ein schwingendes Element, ein Pendel. Je kürzer ein Pendel, desto schneller schwingt es und desto genauer ist die Uhr. Bei einer Cäsium-Atomuhr ist das „Pendel" eine bestimmte elektronische Übergangsfrequenz des Cäsium-133-Atoms; es schwingt rund neun Milliarden Mal pro Sekunde. Exakt 9192631770 Schwingungen dieses kleinen Pendels definieren seit 1967 eine Sekunde. Damit ist Maxwells Forderung, man solle Einheiten auf der Basis atomarer Eigenschaften beruhen lassen, erfüllt. Im Jahr 1967 konnte man die Sekunde auf 14 Stellen hinter dem Komma genau messen. Heute sind es 16 Stellen hinter dem Komma - und damit eine Sekunde Abweichung in 180 Millionen Jahren. Nötig ist diese enorme Präzision für die Satellitennavigation, das "global positioning system" (GPS) aber auch für die physikalische Grundlagenforschung. Interessant ist dabei, dass bei gleichbleibender Definition die Realisierung der Einheit inzwischen um den Faktor 100 gesteigert werden konnte. Das zeigt, dass diese Art der Definition offen für Innovationen ist.

Genauso ist es beim Meter. Im Jahr 1983 legte man die Lichtgeschwindigkeit im Vakuum zahlenmäßig fest. Mithilfe dieses Zahlenwertes und einer gemessenen Zeit wurde der Meter definiert. Heute messen wir kleinste Distanzen bis hinab zum Pikometer. Gleichzeitig sind größte Distanzen zugänglich, etwa die Entfernung zwischen Erde und Mond - und dies mit einer Messunsicherheit von wenigen Zentimetern. Wie bei der Sekunde ist der wissenschaftliche Fortschritt nur durch die technischen Möglichkeiten begrenzt, nicht durch die Definition selbst.

Anders sah es beim Kilogramm aus: Die Einheit der Masse war bis zum 20. Mai 2019 durch das Urkilogramm definiert - jenes Artefakt, das ursprünglich aus der Französischen Revolution kommt und 1889 in der Meterkonvention als internationaler Prototyp des Kilogramms festgelegt wurde.

1889 wurde das Urkilogramm als PlatinIridium-Zylinder hergestellt und in eine Glasvitrine in einen Tresor gelegt. Bis heute wurde es nur dreimal herausgenommen. Denn es ist äußerst wertvoll - alle Masse-Messungen auf der Welt hingen bis zum 20. Mai 2019 an diesem Stück Metall. Bei diesen drei Gelegenheiten wurde es mit seinen besten Kopien verglichen: mit den offiziellen Kopien, die auch in Paris liegen, sowie mit den nationalen Normalen in den nationalen Metrologieinstituten überall auf der Welt. Bei diesen Vergleichsmessungen zeigte sich ein Trend. Zwar bleibt das Kilogrammstück - per definitionem - immer ein Kilogramm. Aber relativ dazu bewegen sich die anderen Normale, und zwar im Bereich von etwa $50 \mu \mathrm{g}$ über 100 Jahre. Das ist sehr wenig, aber für Metrologen eine sehr unbefriedigende Situation. 
Zudem gab es keine Innovationsoffenheit: Die Realisierung des Kilogramms konnte nie genauer werden, als dieses Artefakt und seine Eigenschaften es vorgaben.

Weil sich vermutlich auch das Urkilogramm selber verändert hat, ruht die Einheit der Masse auf unsicherer Basis. Und davon sind auch jene Einheiten betroffen, in deren Definition das Kilogramm eingeht: die Candela (die Einheit der Lichtstärke), das Mol (die Einheit der Stoffmenge) und das Ampere (die Einheit der Stromstärke).

Die bisherige Definition des Ampere beruht auf einer Kraft zwischen zwei stromdurchflossenen Leitern, die unendlich dünn und unendlich lang sind. Das lässt sich experimentell nur näherungsweise umsetzen. Daher wird bereits seit 1990 die Einheit der Stromstärke anders - und zudem sehr viel genauer - realisiert. Grundlage dafür sind Quanteneffekte für den elektrischen Widerstand und die elektrische Spannung sowie die dazugehörigen zwei Konstanten: die von-Klitzing-Konstante und die Josephson-Konstante. Beide beruhen auf Verhältnissen fundamentaler Konstanten, nämlich der Planck-Konstante und der Elektronenladung. Damit bewegte sich allerdings die Realisierung des Ampere außerhalb des SI und seiner Regeln.

Ähnlich war es bei der Temperatur. Sie war definiert durch den absoluten Nullpunkt und den sogenannten Tripelpunkt des Wassers; diejenige Temperatur, bei der Wasser gleichzeitig in festem, flüssigem und gasförmigem Zustand existieren kann. Bei vorgegebenem Luftdruck, hier dem Normaldruck der Atmosphäre, und bei Wasser möglichst ohne jede Verunreinigung ist dies nur bei einer einzigen Temperatur möglich. Auf diesen beiden Fixpunkten baute die Temperaturskala auf, indem die Differenz in genau hundert Teile, Grad Kelvin, unterteilt wird. Also war auch die Definition der Temperatur vollständig unabhängig von allen anderen Einheiten.

Insgesamt basierten also nur Sekunde und Meter auf den Zahlenwerten von Konstanten. Bei den fünf anderen Basiseinheiten (Kilogramm, Ampere, Kelvin, Mol und Candela) gab es eine nicht ideale Situation. Gelänge es, auch sie (und damit das ganze System) quantenbasiert zu machen, würden sie alle innovationsoffen werden. In der Tat ist es nun gelungen, ein kohärentes System zu schaffen, das einheitlich auf Quanten und fundamentalen Naturkonstanten begründet ist.

\section{Naturkonstanten im SI}

Im revidierten SI sind jetzt für alle bisherigen Basiseinheiten die Zahlenwerte von „definierenden Konstanten“ - unter ihnen Naturkon- stanten - festgelegt worden, so wie schon zuvor bei der Sekunde und beim Meter. Auf dieser Basis werden mithilfe der Gleichungen der Physik, also mithilfe des jetzigen Wissens von den physikalischen Zusammenhängen, die Einheiten generiert. Das Ganze soll für alle Zeiten und Kulturen im gesamten Universum (soweit wir es denn kennen) gültig sein, wie Max Planck es gefordert hat.

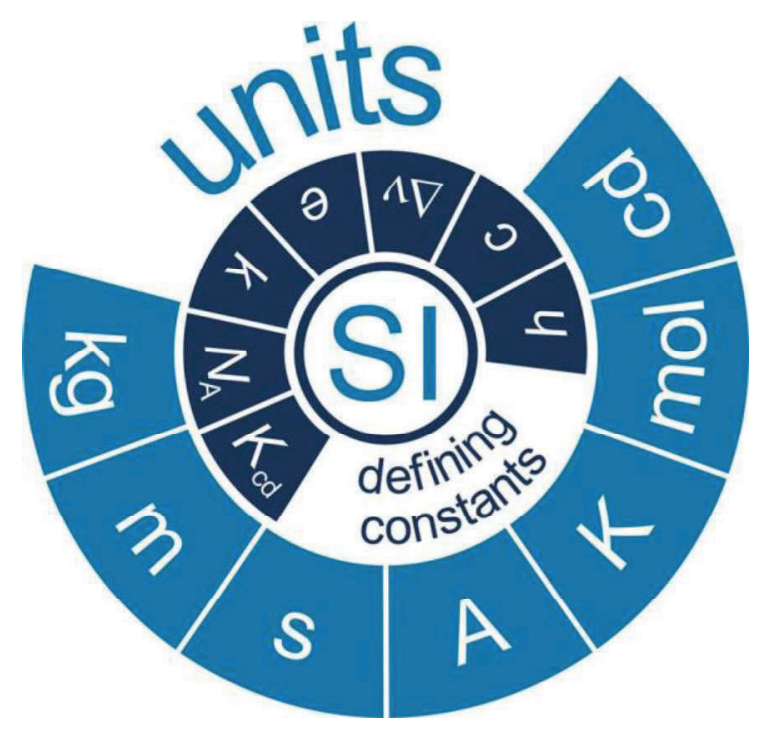

Abb. 5: Das revidierte SI beruht auf einem konsistenten und kohärenten Satz von sieben „definierenden Konstanten".

Im alten Einheitensystem waren Einheiten über Artefakte festgelegt. Mithilfe dieser Einheiten wurden (mit einer gewissen Messunsicherheit) die Naturkonstanten gemessen. Im neuen SI ist es anders herum: Die Werte von Naturkonstanten sind zahlenmäßig festgelegt, und daraus werden die Einheiten abgeleitet. Die Sekunde ist über eine bestimmte Zahl der Schwingungen des „Pendels“ im Cäsium-Atom definiert. Dieselbe Konstante plus die Lichtgeschwindigkeit wird für die Definition des Meters gebraucht. Für das Kilogramm musste noch der numerische Wert einer dritten Konstante festgelegt werden: die Planck-Konstante.

\section{Das Avogadro-Experiment}

Dem Ziel, die Planck-Kontante so genau wie möglich zu ermitteln und schließlich festzulegen, dienten zwei fundamental unterschiedliche experimentelle Herangehensweisen, beides wissenschaftliche Großprojekte, die an verschiedenen nationalen Metrologieinstituten über mehrere Jahrzehnte hinweg betrieben wurden: das Avogadro- und das WattwaagenExperiment. Im Avogadro-Projekt der PTB zusammen mit internationalen Partnern wurde die Planck- 
Konstante mithilfe einer speziell gefertigten Siliziumkugel ermittelt. Sie besteht zu einem sehr hohen Anteil aus einem von drei natürlichen vorkommenden Silizium-Isotopen. Diese Isotopenreinheit garantiert, dass die Masse der Atome in der Kugel sehr genau gemessen werden kann. Kennt man die Masse eines Atoms, lässt sich die Masse der Kilogrammkugel über das Zählen ihrer Atome bestimmen.

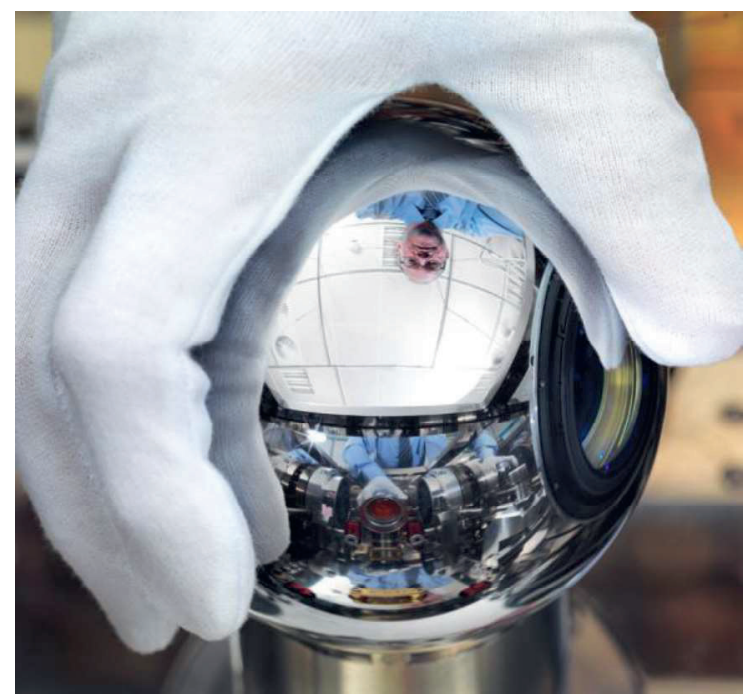

Abb. 6: Das rundeste makroskopische Objekt der Welt: die Siliziumkugel der PTB im internationalen Avogadro-Projekt für ein „neues" Kilogramm und ein „neues" $\mathrm{Mol}$.

Doch die $10^{25}$ Atome lassen sich nur mit indirekten Methoden zählen. Diese nutzen die Tatsache aus, dass Silizium regelmäßige Kristalle bildet. Misst man den Gitterparameter, das Volumen der gesamten Kugel und einige weitere Parameter, lässt sich die Zahl der Atome und die Planck-Konstante ermitteln.

Bei der Volumenmessung der in einer komplexen Fertigungskette hergestellten Si-Kugel mithilfe eines speziellen Kugel-Interferometers lassen sich Abweichungen zwischen Maximum und Minimum auf der Kugeloberfläche von 16 Nanometern ermitteln. Auf die Dimensionen der Erde übertragen, hieße das: Die größten Höhenunterschiede zwischen tiefstem Ozean und höchstem Berg betrügen nur zwei Meter. Und dies wird mit einer Genauigkeit von zwei oder drei Millimetern gemessen. Das liegt an der Grenze der heutigen Längenmesstechnik. Als nächster Schritt wird mit einer der besten Waagen der Welt die Masse der gesamten Kugel ermittelt. Die Masse der Kugel geteilt durch die Zahl der Atome ergibt die Masse eines einzelnen Atoms - und letztlich die Planck-Konstante.
Keiner dieser Werte lässt sich auf direktem Wege messen. Die gewünschten Konstanten (neben der Planck- auch die AvogadroKonstante für die Einheit der Stoffmenge, Mol) werden rechnerisch aus den Messungen ermittelt. Wird, so wie im revidierten SI, der Zahlenwert der Planck- (Avogadro-) Konstanten festgelegt, realisiert man über das Abzählen der Atome in der Siliziumkugel die Einheit der Masse (Kilogramm) und der Stoffmenge (Mol).

\section{Das Wattwaagen-Experiment}

Eine zweite Methode auf dem Weg zu einem neuen Kilogramm ist die sogenannte Wattoder Kibble-Waage. Das Prinzip ist relativ einfach: Auf der einen Seite der Waage liegt das zu messende Massestück. Die entsprechende Gewichtskraft wird auf der anderen Seite der Waage mit einer elektromagnetischen Kraft kompensiert, wobei die elektrischen Messgrößen mit elektrischen Quantennormale bestimmt werden. Als Ergebnis erhält man eine Gleichung, die den Zusammenhang zwischen Masse und Planck-Konstante herstellt. Legt man den Zahlenwert der Planck-Konstanten fest, so kann man mit einer solchen Waage das Kilogramm realisieren. Die Methode ist vollständig anders als in dem SiliziumkugelProjekt. Über den Vergleich der beiden unterschiedlichen Methoden wird sichergestellt, dass die zugrundeliegenden physikalischen Gleichungen mit der geforderten Genauigkeit übereinstimmen.

\section{Elektronen zählen}

Elektrischer Strom besteht aus einzelnen Elektronen, die in einem Zeitintervall durch einen Leiter fließen. Gelingt es, einzelne Elektronen mit einer bestimmten Frequenz durch einen Leiter zu schicken und diese Frequenz zu messen, dann lässt sich über den festgelegten Zahlenwert der Elektronenladung der Strom definieren und messen. Konzeptionell einfach, jedoch in der Praxis sehr komplex. Zur Kontrolle der Elektronen verwendet man Quantenpunkte: Nanostrukturen, mit denen sich einzelne Elektronen einfangen und einzeln hindurchschieben lassen; sie werden auch "Elektronenpumpen" genannt. Elektronen sind "Quantenteilchen", bei denen man nie mit hundertprozentiger Genauigkeit wissen kann, ob genau ein Elektron "gepumpt" wurde. Daher braucht man Detektoren, die die einzelnen Elektronen beim Verlassen der Pumpe nachweisen können. Schaltet man drei Pumpen und Nachweissysteme hintereinander, verzählt man sich bei zehn Millionen gepumpter Elektronen nur etwa zweimal und kann die Einheit Ampere auf sieben Stellen hinter dem Komma genau realisieren. 
Genauso wie bei diesen vier Basiseinheiten (Sekunde, Meter, Kilogramm, Ampere) funktioniert es auch bei den drei übrigen: Durch Festlegung des Zahlenwertes einer zugehörigen Naturkonstante lassen sich Einheiten definieren. Beim Mol ist es die Avogadro-Kontante, beim Kelvin die Boltzmann-Konstante und bei der Candela das photometrische Strahlungsäquivalent.

\section{Eigenschaften des SI}

Dem revidierten SI liegt ein fundamental neues Konzept zugrunde, das viele Vorteile bietet: Es garantiert Langzeitstabilität. Die Konstanten behalten für alle Zeit den festgelegten Wert und ändern sich nicht mehr. Ein weiteres Charakteristikum ist, dass man im Allgemeinen mehr als eine Konstante braucht, um eine Einheit zu realisieren: Beim Ampere sind es zwei (Elektronenladung und Frequenz des Hyperfeinstrukturüberganges), beim Kilogramm drei (Frequenz des Hyperfeinstrukturüberganges, Lichtgeschwindigkeit und Planck-Konstante). Meist stehen zur Realisierung der Einheiten unterschiedliche Messmethoden zur Verfügung, die helfen, die Ergebnisse zu kontrollieren. Beim Kilogramm sind es das Abzählen von Atomen in der Siliziumkristallkugel und die Watt- oder Kibblewaage anstelle des einen Urkilogrammstückes in Paris. Die Wissenschaftler sind vollständig unabhängig von bestimmten Orten: Jeder in einem Labor überall auf der Welt (oder auf einem anderen Stern) kann ein Experiment aufbauen, um eine Einheit zu realisieren. Eine Einheit ist nicht nur mehr an einem einzelnen Messpunkt definiert, also bei genau einem Kilogramm für die Masse oder durch die zwei Punkte für die Definition der Temperaturskala, den absoluten Nullpunkt und den Tripelpunkt des Wassers. Durch das Fertigen kleinerer Siliziumkugeln oder von Silizium-Nanokristallen sowie mithilfe von Wattwaagen lässt sich das Kilogramm über weite Bereiche der Masseskala direkt realisieren. Analog ist es insbesondere auch bei der Temperatur, wo man verschiedene Gleichungen heranziehen und so die ganze Temperaturskala abdecken kann. Schließlich sind die elektrischen Einheiten nicht mehr isoliert in ihrer eigenen Quantenwelt, sondern in das ganze System integriert. Alle Einheiten sind in der Quantenwelt angekommen, ihre Konsistenz gesichert.

\section{Offen für Innovationen}

Ein entscheidender Vorteil des revidierten Systems ist die Tatsache, dass sich durch verbesserte Experimente die Einheiten immer besser realisieren lassen, ohne die Definition zu ändern. Nicht mehr Artefakte, sondern nur noch die Quantennatur der Welt limitiert die Experimente. Während sich vorher eine Masse auf höchstens acht Stellen hinter dem Komma genau bestimmen ließ, weil der KilogrammPrototyp in dieser Größenordnung schwankte, ist diese Grenze mit den neuen Definitionen aufgehoben. Prinzipiell wäre sogar eine bis auf ein Atom genaue Massenbestimmung, also mit etwa 25 Stellen hinter dem Komma, möglich. Das heißt, die Definition lässt viel Freiheit für Verbesserungen.

Beim Ampere begrenzte die alte Definition (Kraft zwischen zwei Leiterbahnen) die Realisierung auf sieben Stellen hinter dem Komma. Jetzt ist die prinzipielle Grenze im neuen System durch die sehr kleine Ladung des Elektrons gegeben, bei etwa $10^{-19}$ Coulomb. So lässt sich im Prinzip, wenn Elektronen pro Sekunde abgezählt werden und man sich nur um ein Elektron verzählt, ein Ampere auf 19 Stellen hinter dem Komma genau messen.

Die Limitierung liegt nicht mehr in den Definitionen, sondern in den Gesetzen der Quantenphysik, in der Tatsache, dass offensichtlich die Messgrößen nicht unendlich klein werden können, sondern in der Natur in kleinsten Paketen, den Quanten vorkommen. Das ist eine bahnbrechende Verbesserung und in diesem Sinne eine enorme Veränderung.

Allerdings sollte einen Tag nach Inkrafttreten des revidierten SI ein Kilogramm, etwa bei Einkäufen im Supermarkt, noch genauso schwer sein wie am Tag davor. Es durfte im Alltagsleben keine Änderungen geben. Daher sind die jetzt festgelegten Werte der Konstanten so „krumm". Für "gerade" Werte der Konstanten hätten zwangsläufig die Länge eines Meters, die Masse eines Kilogrammes usw. verändert werden müssen. Zudem musste sichergestellt werden, dass sich diese Konstanten mit einer Genauigkeit messen lassen, die für die Hightech-Messindustrie gebraucht wird, um die Einheiten zu realisieren.

Es war die Arbeit vieler Metrologieinstitute und hunderter Metrologen auf der ganzen Welt. Was zum Teil einfach aussieht, beruht auf Hochpräzisionsexperimenten und jahrelangen Entwicklungen. Erst 2017, genau 117 Jahre nach der wegweisenden Idee von Max Planck, waren die Voraussetzungen erfüllt, um das letzte Artefakt loszuwerden. Im Herbst 2017 sprach ein Expertengremium, das Internationale Komitee für Maße und Gewichte (CIPM), die Empfehlung aus, das Einheitensystem grundlegend zu revidieren. Ein Jahr später, am 16. November 2018, beschloss die Generalkonferenz für Maße und Gewichte (CGPM) die Revision. Am 20. Mai 2019 trat mit dem revidierten SI ein neues System der Einheiten in Kraft, das Langzeitstabilität, universelle Realisierbar- 
keit, Genauigkeit, die mit dem technologischen Fortschritt mitwächst, sowie Innovation in Wirtschaft und Technik garantiert.

An einer Stelle wurde nicht das gemacht, was Max Planck gefordert hatte, nämlich durchgehend Fundamentalkonstanten zu verwenden: Die Definition der Sekunde beruht weiterhin auf der Eigenschaft eines bestimmten Atoms. Dieses, nämlich Cäsium, wurde lediglich aus pragmatischen experimentellen Gründen gewählt. Diese Inkonsequenz hat gute Gründe: Die so definierte Sekunde lässt sich genauer als jede andere Einheit messen: auf 16 Stellen hinterm Komma. Und es gibt schon Uhren, die auf 18 Stellen hinterm Komma genau ticken. Allerdings beruhen sie auf Pendeln in anderen Atomen, die bis zu hunderttausendmal schneller schwingen als die im Cäsium-Atom. Im Jahr 2026 könnte mithilfe dieser sogenannten optischen Atomuhren die Sekunde neu definiert werden. Bereits jetzt werden sie in der Praxis verwendet. Hebt man eine solche Uhr im Gravitationsfeld der Erde um einen Zentimeter an, dann geht sie nach Einsteins Relativitätstheorie um $10^{-18}$ Sekunden anders. Damit ist eine optische Uhr auch ein Detektor für das Gravitationsfeld der Erde, mit dem sich unterirdische Lager oder Wasserströme detektieren lassen. Man kann mit innen im Detail sehen, wie das Eis in Grönland aufgrund der Erderwärmung schmilzt, oder beim Tunnelbau von zwei Seiten z. B. durch die Alpen sicherstellen, dass sich die beiden Bohrtrupps auf Zentimeter genau aufeinander zubewegen. Es gab also gute Gründe, diese Definition aufrechtzuerhalten. Sie ist praktikabel, hochpräzise und sehr gut wenn auch nicht ganz im Sinne des Theoretikers.

\section{Konstante Konstanten?}

Sind diese Konstanten, die wir jetzt definiert und festgelegt haben, physikalisch gesehen wirklich konstant, oder ändern sie sich im Laufe der Zeit? Genau dies sagen einige physikalische Theorien voraus, die darauf zielen, eine vereinheitlichte Theorie der Physik zu entwickeln, also die elektroschwache, die starke sowie die gravitative Wechselwirkung zu vereinheitlichen.

Dazu gibt es Experimente, insbesondere zur sogenannten Sommerfeld'schen Feinstrukturkonstante, einer Kombination von Elektronenladung, Lichtgeschwindigkeit und PlanckKonstante. Die weltweit genauesten Experimente an der PTB mit den besten verfügbaren Uhren zeigen, dass die jährlichen Änderungen - wenn sie überhaupt vorhanden sind - höchstens an der 18. Stelle hinterm Komma liegen. Sie sind also auf jeden Fall so klein, dass sie die Präzision der Realisierung der Einheiten im revidierten System zumindest auf sehr lange Zeit nicht merkbar beeinflussen werden - nicht nur bei den Genauigkeiten, die im alläglichen Leben gefragt sind, sondern auch für höchste Präzision, wie sie in Hightech-Bereichen der Industrie und in der Wissenschaft gefordert sind.

Die Menschheit hat einen jahrtausendelangen Weg zurückgelegt: von den ersten Artefakten bis hin zu einem System, das die wohl höchste erreichbare Abstraktionsstufe darstellt, um ein Einheitensystem zu generieren, das nach den Kriterien der Französischen Revolution universell ist - nicht nur auf der Erde, sondern auch im gesamten uns bekannten Universum, entsprechend der Vision von Max Planck. Für alle Zeiten, für alle Culturen!

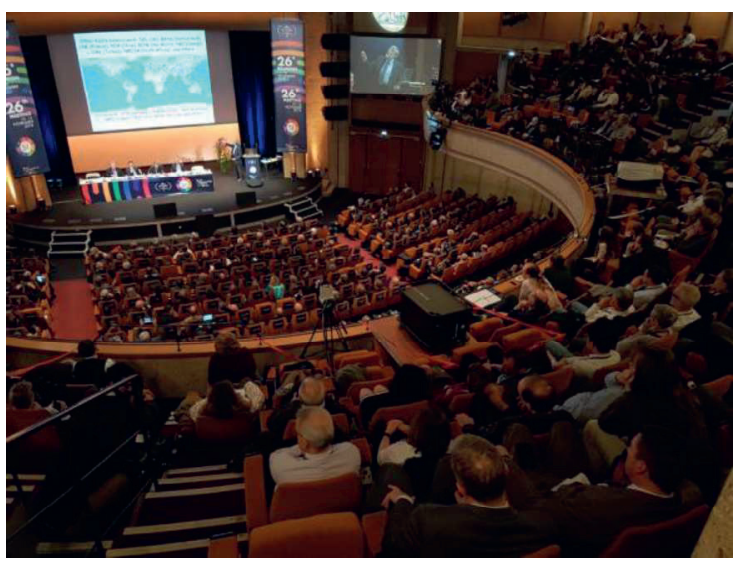

Abb. 8: Bei der Generalkonferenz für Maße und Gewichte in Versailles beschlossen die Staaten der Meterkonvention am 16. November 2018 einstimmig das "neue" SI. (Quelle: BIPM)

\section{Literatur}

[1] Decisions of the 26th meeting of the General Conference of Weights and Measures, Versailles, 13-16 November 2018, www.bipm.org/en/measurement-units/rev-si/

[2] The International System of Units, 9th edition, Bureau international des poids et mesures www.bipm.org/en/publications/si-brochure/

[3] Das neue Internationale Einheitensystem (SI), PTB-Infoblatt, November 2017, www.ptb.de 